\title{
Simple prediction of FSS radome transmission characteristics using an FSS equivalent circuit model
}

\author{
Yeong-Chul Chung ${ }^{1}$, Kyung-Won Lee ${ }^{1 \mathrm{a})}$, Ic-Pyo Hong ${ }^{2}$, \\ Myung-Gun Lee ${ }^{3}$, Heoung-Jae Chun ${ }^{4}$, and Jong-Gwan Yook ${ }^{1}$ \\ ${ }^{1}$ Dept. of Electrical and Electronic Engineering, Yonsei University, \\ 134, Shinchon Dong, Sedaemun Gu, Seoul Korea \\ ${ }^{2}$ Dept. of Information and Communication Engineering, Kongju University, \\ 330-717, Cheonan Korea \\ ${ }^{3}$ Agency for Defense Development, Daejeon Korea \\ ${ }^{4}$ Dept. of Mechanical Engineering, Yonsei University, \\ 134, Shinchon Dong, Sedaemun Gu, Seoul Korea \\ a)lkwl88@yonsei.ac.kr
}

Abstract: We propose a simple method for predicting the characteristics of Frequency Selective Surface (FSS) radome using an equivalent circuit model. The required material properties, such as the permittivity and permeability of the FSS, were extracted from a FSS equivalent circuit model using a transmission and reflection method. These parameters were then used to calculate the transmission characteristics of FSS radome. We designed a sandwich radome using a square loop FSS for a $23 \mathrm{GHz}$ band application. In order to demonstrate the validity of our approach, we also performed a simulation to obtain the characteristics of the FSS radome using commercial full-wave software. The results from the two approaches are in good agreement. The method proposed herein could be used for the simple and rapid calculation of the design and fabrication of a FSS radome. The FSS radome is that a square loop FSS that has a stop band at $23 \mathrm{GHz}$ is insulated between two dielectric materials. A simple analysis of a FSS radome requires the material constants, the permittivity and permeability of the square loop FSS, which we extracted using transmission/reflection. Using the material constants derived from a FSS equivalent circuit model, the characteristics of the FSS radome were calculated for a simple 2-D case. The results were verified using a commercial full-wave three-dimensional electromagnetic wave simulator. The resulting characteristics of the FSS radome agreed with those obtained using a full-wave simulation. We show herein that the characteristics of a FSS radome can be analyzed and predicted with relative ease using an approximate FSS to achieve a simple 2-D construction. Our method can be applied to the analysis of FSS radome for use with various communication systems 
and electrical instruments.

Keywords: FSS radome, square loop FSS, equivalent circuit model, boundary value method, transmission/reflection (T/R) method

Classification: Electromagnetic theory

\section{References}

[1] B. A. Munk, Frequency Selective Surface: Theory and Design, Wiley Interscience, New York, 2000.D.D.L. Chung, Materials for electromagnetic interference shielding. J Mater Eng Perform 9 3, pp. 350-354, 2000.

[2] N. Marcutvitz, Waveguide Handbook, Dover Publications, New York, 1965.

[3] R. J. Langley and E. A. Parker, "Equivalent circuit model for arrays of square loops," Electron. Lett., vol. 18, no. 7, pp. 294-296, April 1982.

[4] C. K. Lee and R. J. Langley, "Equivalent-circuit models for frequency selective surfaces at oblique angles of incidence," IEE Proc. $H$ - Microwaves Optics and Antennas, vol. 132, pp. 395-399, Oct. 1985.

[5] Y. Kuzuoglu, "Design of the Square Loop Frequency Selective Surfaces with Particle Swarm Optimaization via the Equivalent Circuit Model," Radioengineering, vol. 18, no. 2, June 2009.

[6] J. Baker-Jarvis, "Transmission/Reflection and Short-Circuit line Permittivity Measurement," NIST Report, July 1990.

[7] J. Baker-Jarvis, E. J. Vanzura, and W. A. Kissick, "Improved Technique for Determining Complex Permittivity with the Transmission/Reflection Method," IEEE Trans. Microw. Theory Tech., vol. 38, no. 8, pp. 10961103, Aug. 1990.

[8] D. J. Kozakoff, "Analysis of radome-enclosed antennas," Artech House, Inc., 1997.

[9] A. Kedar and U. K. Revankar, "Parametric study of flat sandwich multilayer radome," Progress in Electromagnetics Research, PIER 66, pp. 253$265,2006$.

[10] H. Cory and C. Zach, "Wave propagation in metamaterial multi-layered structures," Microw. Opt. Technol. Lett., vol. 40, no. 6, March 2004.

\section{Introduction}

Frequency Selective Surfaces (FSSs) are periodic surfaces that have identical elements arranged in one or two dimensional arrays. FSSs have recently been widely used in spatial filters in radomes for antenna and radar systems, microwave absorbers, and in sub-reflectors of dual antennas in communication systems. The element patterns of FSSs are determined by the applications of the system concerned and the desired frequency response [1]. There has been a great deal of research on the analysis and design of the FSS element using analytical or numerical electromagnetic methods. Among the possible methods, the equivalent circuit model approach to the design of FSSs is very popular because of its simplicity and the ease with which it can be understood. The equivalent circuit model is based on the theory proposed by Marcuvitz [2], who developed the initial expression for the periodic gratings. Langley and Parker [3] derived equivalent circuit models for 
square loop FSSs, including gridded square loops and double square loops. Lee and Langley [4] also proposed the improved equivalent circuit model for oblique incidence. The transmission/reflection (T/R) method is a wellknown method for extracting material constants from an arbitrary material and the working principles for transmission/reflection have been analyzed systematically by Baker-Jarvis [6, 7].

There are two types of method commonly used in the theoretical design of a multilayer radome, namely the boundary value solution [8] and the recursive method. Kedar and Revankar [9] presented a method for parametric analysis for multilayer radome design based on the boundary value solution technique, and Cory and Zach [10] used a recursive method to calculate the reflection and the transmission coefficients for multilayered structures. We show herein that the FSS structure can be represented in terms of a simple layer by extracting the material constant of the FSS structure using an equivalent circuit model, and then the 3-layer structure of the FSS radome can be also predicted relatively easily.

\section{Square loop FSS and extraction of material constant}

In our FSS radome design, we used a square loop FSS that had a stop band at $23 \mathrm{GHz}$. The geometrical dimensions of the square loop FSS shown in Fig. 1 (a) are given by p, d, and w. This FSS is equivalent to a simple L-C circuit model and can be represented by Eq. (1a), in which $\mathrm{Y}$ is the calculated admittance of the square loop using an equivalent circuit and $\tau$ is the transmission coefficient. $\mathrm{Y}$ is represented by $\mathrm{C}$ and L as per Eq. (1b) [3, $4,5]$.

$$
\begin{gathered}
|\tau|^{2}=\frac{4}{4+|Y|^{2}} \\
Y=\left(j \omega L+\frac{1}{j \omega C}\right)^{-1}
\end{gathered}
$$

where $\mathrm{L}$ and $\mathrm{C}$ are given as:

$$
\begin{gathered}
\omega L=F(p, 2 w, \lambda) \cdot\left(\frac{d}{p}\right) \\
\omega C=4 \cdot F(p, g, \lambda) \cdot\left(\frac{d}{\varepsilon_{r} p}\right)
\end{gathered}
$$

where p, w, d and g are given by Fig. 1 (a) and $\omega$ is angular frequency and $\lambda$ is wavelength and $\varepsilon_{r}$ is dielectric constant of FSS substrate $\left(\varepsilon_{r}=1\right.$ in this design). $F$ function of the Eq. (2) can be defined by the equations of the strip grating (see [2] for more details).

When transmission coefficient $(\tau)$ is a + jb, Eq. (5) can be derived using relation of Eq. (3) and (4). Therefore, The FSS characteristics shown in Figs. 1 (b) and (c) can be predicted.

$$
\begin{gathered}
|\rho|^{2}+|\tau|^{2}=1 \\
\rho+1=\tau
\end{gathered}
$$




$$
\begin{gathered}
\tau=|\tau| \angle \tan ^{-1}\left(\frac{b}{a}\right) \\
\rho=|\rho| \angle \tan ^{-1}\left(\frac{b}{a-1}\right) \\
\frac{b}{a-1}=-\frac{2}{\operatorname{Im}(Y)} \\
\frac{b}{a}=\frac{\operatorname{Im}(Y)}{2}
\end{gathered}
$$

Fig. 1 (b) and Fig. 1 (c) show the S-parameters and phase, respectively, obtained from the calculated values from the equivalent circuit model for a square grid FSS compared to the result obtained by full-wave simulation using a commercial simulator. All the simulations have been compared to the result obtained by full-wave simulation using Ansoft HFSS ver 12. Figs. 1 (b) and (c) illustrate the good agreement between these two approaches.

In order to extract the material parameters of permittivity and permeability of the FSS, we used the transmission/reflection (T/R) method. This is a well-known method, which requires four S-parameters (S11, S21, S12,
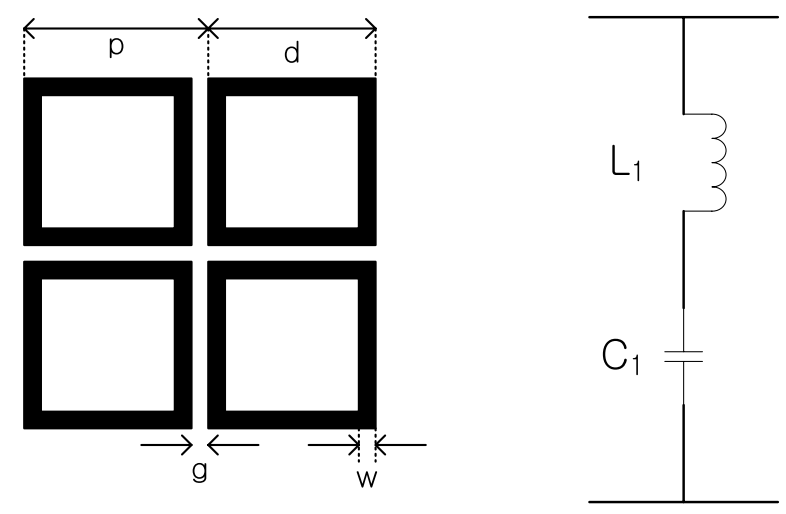

(a) Square Loop FSS and Equivalent Circuit Model

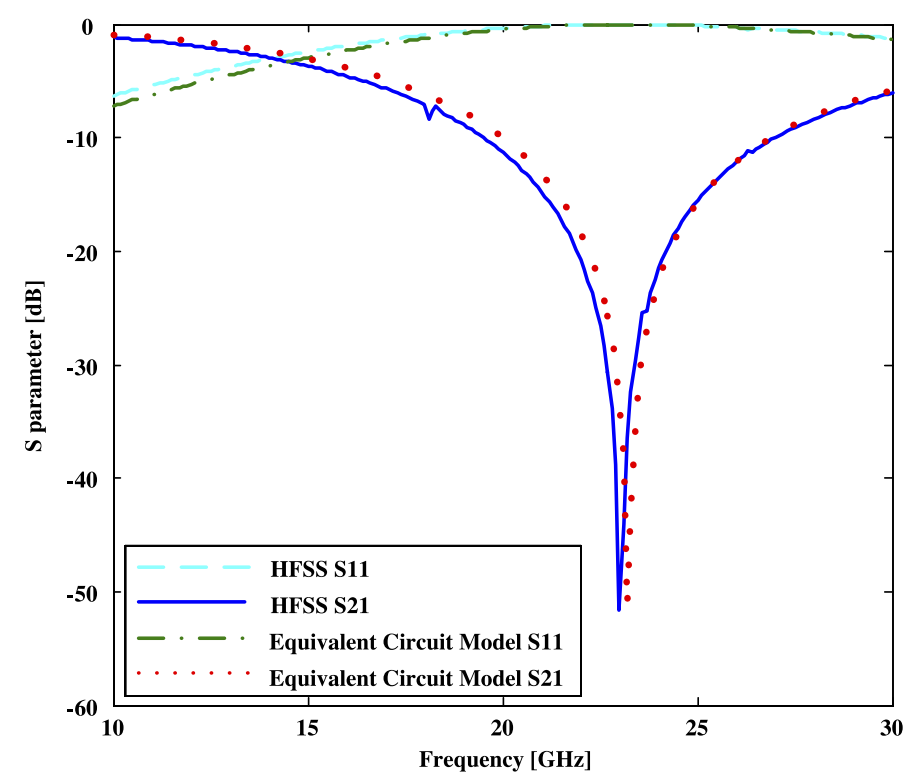

(b) Magnitude 


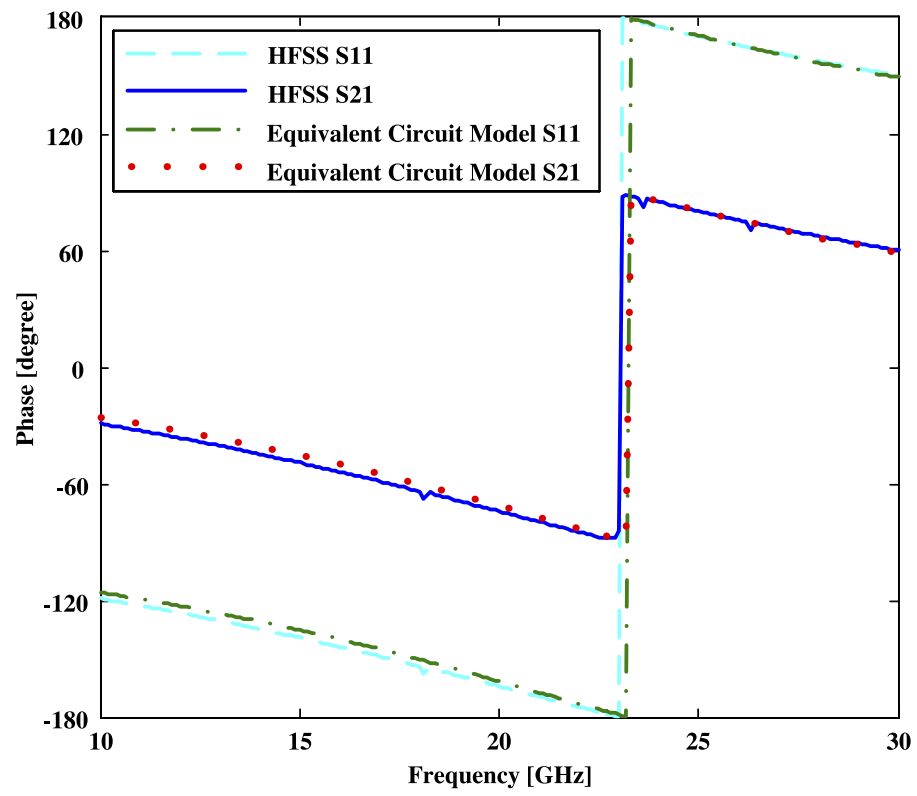

(c) Phase

Fig. 1. Construction and Characteristic of a Square Loop FSS

S22) in order to extract the material constants. Although S22 and S12 are unknown, we assume that S11 and S21 can be used in their place, because of the reciprocity of the properties of the FSS design. Fig. 2 shows the extracted complex material parameters of the square loop FSS. Fig. 2 (a) represents the complex permittivity. The real value of the extracted permittivity is very high at the resonant frequency of the FSS $(23 \mathrm{GHz})$. The imaginary value is approximately zero, but shows a small peak at the resonant frequency, which implies that the FSS used in the analysis has no magnetic property.

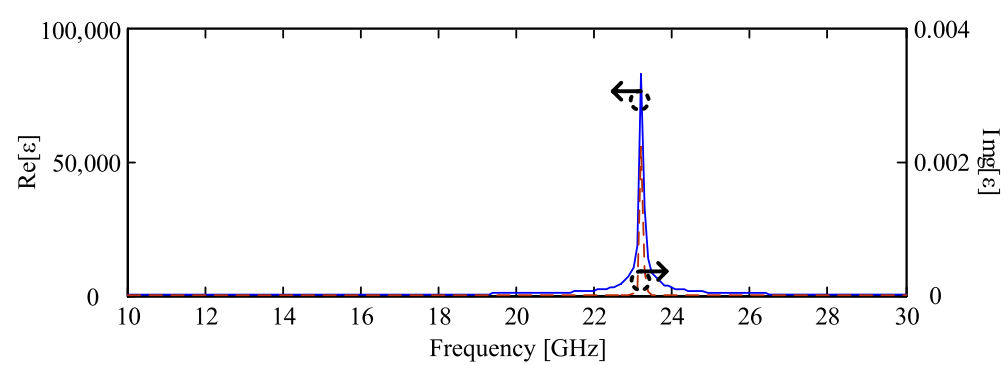

(a) Complex Permittivity

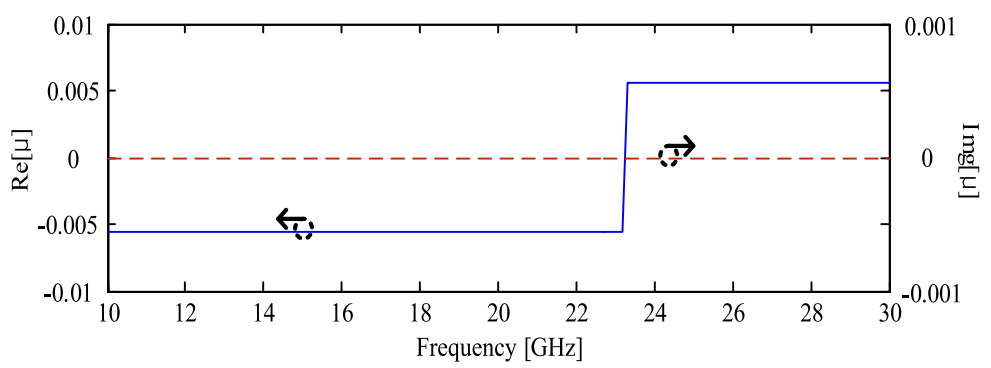

(b) Complex Permeability 
Fig. 2 (b) depicts the complex permeability. The real value changes sign (from negative to positive) at $23 \mathrm{GHz}$ due to the $180^{\circ}$ phase reversal of S11, but it is approximately zero at all frequencies. The imaginary permeability is also approximately zero. This makes sense because FSSs are pure conductors without any magnetic component.

\section{Analysis of FSS radome}

For the simple analysis of an FSS radome such as that shown in Fig. 3 (a), the FSS structure can be represented by a simple layer that has the required frequency-dependent characteristics. Fig. 3 (a) may therefore be modeled using a 3-layer structure that has an $\varepsilon$ (f) layer in place of the FSS (Fig. 3 (b)). We have assumed the same dielectric material for the sandwich layers of the FSS, a permittivity of 3.3 , and a lossless material. The sandwiched layer of Fig. 3(b), the FSS layer, is the frequency dependent material with the complex permittivity and permeability depicted in Fig. 2.

In order to demonstrate the validity of the method proposed in this letter, we have performed comparisons between the boundary value method and the method of full-wave simulation using commercial software. Fig. 3 (c) shows that the results obtained from the boundary value method agree very well with the results obtained from the full-wave simulation using the same transmission and reflection characteristics for the FSS radome. This result shows that the transmission characteristics of the FSS radome may be predicted quite easily, without the need for a full-wave simulation, provided that the exact equivalent circuit model of the FSS itself is available.

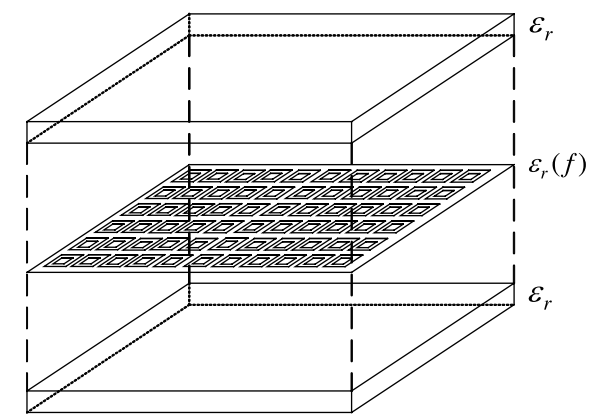

(a) FSS radome structure

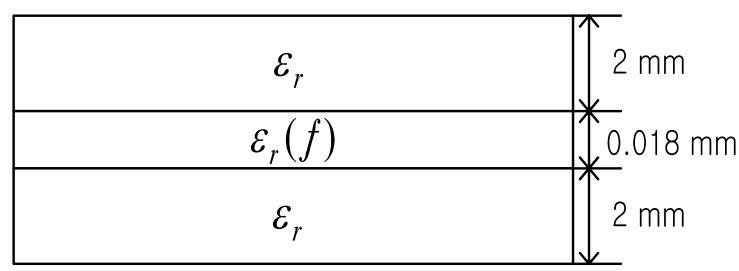

(b) Simple equivalent model(3-layer) 


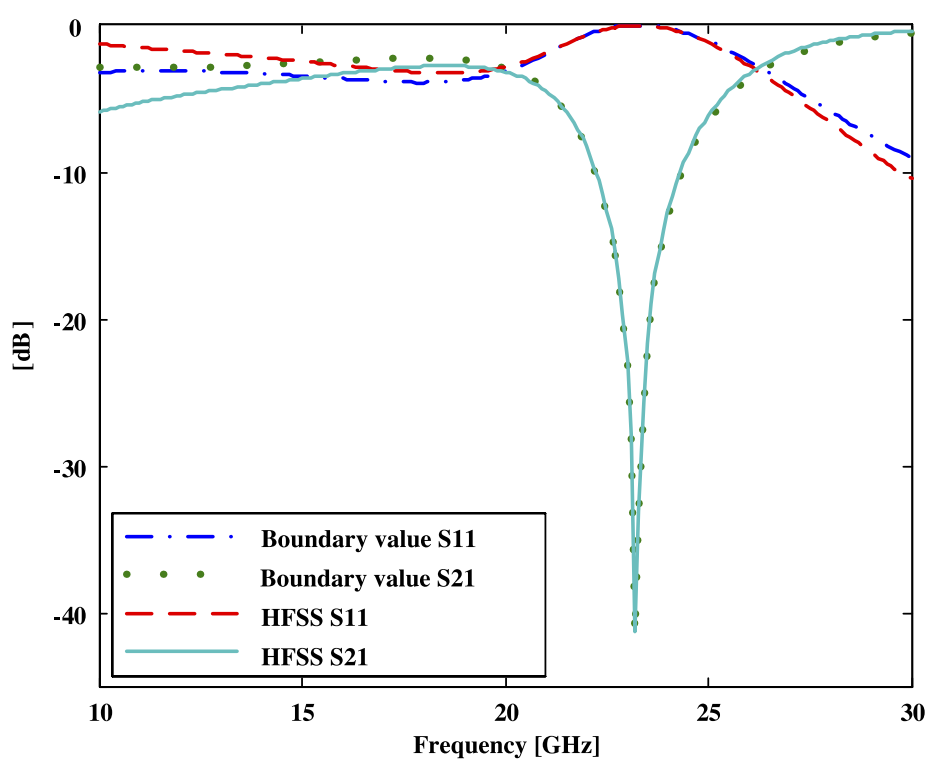

(c) Comparison between results

Fig. 3. FSS radome construction and comparison between analytical result and full-wave simulation

\section{Conclusion}

Herein, we have proposed a simple design method for FSS radomes using an FSS equivalent circuit model. Using this method, we derived the required material parameters of permittivity and permeability to model the equivalent multilayer radome structure. The results show that an FSS radome can be modeled using simple 3-layer structures and that its transmission characteristics can be predicted relatively easily without consideration of a full-wave simulation. These results were compared with those from a full-wave simulation using commercial software (HFSS) and the results show good agreement. We have shown that FSS radomes can be analyzed with ease using the FSS equivalent circuit model. We believe that the method proposed herein could be applied to the design of FSS radomes for use with various communication systems and on aircraft.

\section{Acknowledgments}

This work was supported by the Low Observable Technology Research Center Program of the Defense Acquisition Program Administration and the Agency for Defense Development of Republic of Korea. 